\title{
Identification of Breast Cancer Stem Cells Using a Newly Developed Long-acting Fluorescence Probe, C5S-A, Targeting ALDH1A1
}

\author{
AOI OKAMOTO ${ }^{1}$, YOHEI FUNAKOSHI ${ }^{2}$, MASAHIRO OE ${ }^{3}$, RYO TAKAI $^{4}$, HIROTAKA SUTO ${ }^{2}$, \\ YOSHIAKI NAGATANI ${ }^{2}$, MEIKO NISHIMURA ${ }^{2}$, YOSHINORI IMAMURA ${ }^{2}$, TOMONARI KUNIHISA ${ }^{1}$, \\ NAOMI KIYOTA ${ }^{2,5}$, KOJI MIKI ${ }^{3}$, KOUICHI OHE ${ }^{3}$, HIROKAZU TANINO ${ }^{1 *}$ and HIRONOBU MINAMI ${ }^{2,5}$ \\ ${ }^{1}$ Division of Breast and Endocrine Surgery, Department of Surgery, \\ Kobe University Hospital and Graduate School of Medicine, Kobe, Japan; \\ ${ }^{2}$ Division of Medical Oncology/Hematology, Department of Medicine, \\ Kobe University Hospital and Graduate School of Medicine, Kobe, Japan; \\ ${ }^{3}$ Department of Energy and Hydrocarbon Chemistry, Graduate School of Engineering, \\ Kyoto University, Kyoto, Japan; \\ ${ }^{4}$ Division of Gastrointestinal Surgery, Department of Surgery, \\ Kobe University Hospital and Graduate School of Medicine, Kobe, Japan; \\ ${ }^{5}$ Cancer Center, Kobe University Hospital, Kobe, Japan
}

\begin{abstract}
Background/Aim: Aldehyde dehydrogenase (ALDH) IA1 is a well-known marker for cancer stem cells (CSCs), characterized by self-renewal capacity and multidrug resistance in breast cancer. We developed a near-infrared turn-on fluorescence probe for ALDH1A1, C5S-A, which is suitable for observing and analyzing viable cells. Here, we demonstrated the utility of $C 5 S$ - $A$ in $C S C$ research using breast cancer cell lines. Materials and Methods: To evaluate concordance between C5S-A and conventional stem cell markers, breast cancer cells sorted for ALDEFLUOR-positive cells and for $C D 44^{+} / C D 24^{-}$ cell populations were stained with C5S-A. Tumorigenicity of C5S-A-positive cells was examined by mammosphere formation assay and subcutaneous transplantation to immunodeficient
\end{abstract}

\#Current affiliation: Division of Breast Surgery, Department of Surgery, Wakayama Medical University, Wakayama, Japan.

Correspondence to: Yohei Funakoshi, MD, Ph.D., Division of Medical Oncology/Hematology, Department of Medicine, Kobe University Hospital and Graduate School of Medicine, 7-5-2 Kusunoki-cho, Chuo-ku, Kobe 650-0017, Japan. Tel: +81 783825820, Fax: +81 783825821, e-mail: yohei@med.kobe-u.ac.jp

Key Words: Near-infrared turn-on fluorescence probe, C5S-A, activity of aldehyde dehydrogenase, breast cancer, stem cells.

This article is an open access article distributed under the terms an conditions of the Creative Commons Attribution (CC BY-NC-ND) 4.0 international license (https://creativecommons.org/licenses/by-nc-nd/4.0), mice. Additionally, to determine how long fluorescence from a single staining remained observable, we cultured breast cancer cells for 5 days after C5S-A staining. We then evaluated whether C5S-A-positive cells possessed resistance to cytotoxic drugs by chronological imaging. Results: C5S-A staining showed good concordance with conventional breast CSC markers, and good utility for research into CSC characteristics in breast cancer cell lines, including tumorigenesis. Additionally, C5S-A was observable for more than 3 days with a single staining. Using this property, we then confirmed that C5S-A-positive cells possessed resistance to cytotoxic drugs, which is one of the characteristics of CSCs. Conclusion: We showed that C5S-A is suitable for CSC research using breast cancer cell lines, and confirmed its utility in observing cells over time.

Cancer stem cells (CSCs) are a subpopulation of tumor cells that have self-renewal capacity and differentiation potential, and contribute to tumor malignancy, including to recurrence, metastasis and multidrug resistance (1). These characteristics make CSCs an important research and therapeutic target, and several biomarkers for these cells have now been identified (2).

Increased activity of aldehyde dehydrogenase 1 (ALDH1) is reported to be one such marker of CSCs (3). ALDH1 is a nicotinamide-adenine dinucleotide phosphate-dependent oxidase which mediates the oxidation of intracellular aldehydes into carboxylic acids. This enzyme has various biological functions, including cellular detoxification of cigarette smoke, alcohol, and cytotoxic drugs (4, 5). Importantly, high ALDH1 activity is associated with strong 


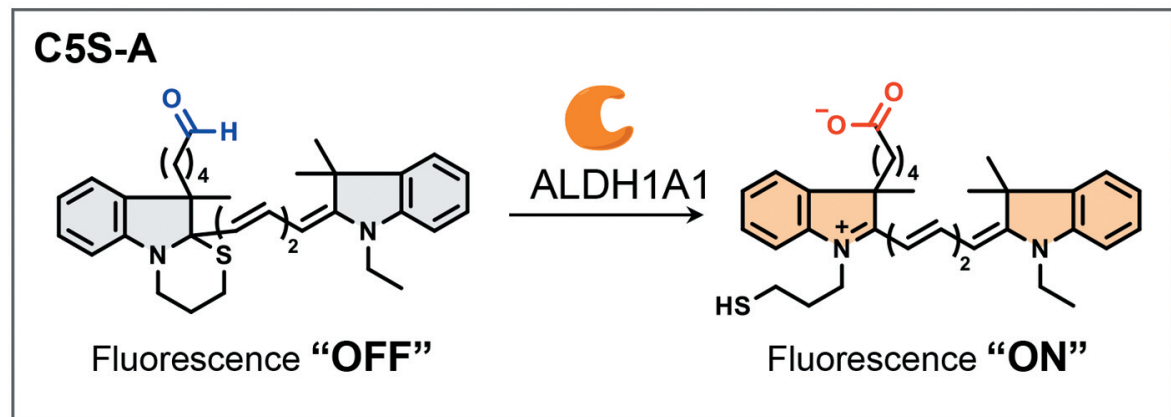

Figure 1. Schematic illustration of cancer stem cell imaging using C5S-A. Structure of the aldehyde dehydrogenase 1 A1 (ALDH1A1)-responsive turn-on near-infrared fluorescence probe C5S-A and its turn-on mechanism.

tumorigenic capabilities in in vivo and in vitro experiments $(6,7)$, and is an independent predictor of early metastasis and reduced survival of patients with breast cancer $(3,6)$. Furthermore, it has been demonstrated that resistance to cytotoxic drugs is related to the transcriptional activation of ALDH1 expression (8). ALDH1 is therefore considered to be an important CSC marker, especially in breast cancer.

Among ALDH1 isoforms, ALDH1A1 is considered a key isoform for CSCs (9). To identify and isolate cells positive for ALDH1A1, we developed a near-infrared (NIR) turn-on fluorescence probe, C5S-A (Figure 1), which is targeted to ALDH1A1 (10). Use of C5S-A has a number of advantages over green-fluorescence ALDEFLUOR, the conventional ALDH1 sensor commonly used in the detection of ALDH1positive cells. In particular, the signal/noise ratio of 8.3 for C5S$\mathrm{A}$ is substantially higher than that of ALDEFLUOR (1.9), and C5S-A shows high contrast in CSC imaging (10). Additionally, while the absorption and emission maxima of ALDEFLUOR are in the ultraviolet to visible region $(350-600 \mathrm{~nm}), \mathrm{C} 5 \mathrm{~S}-\mathrm{A}$ works in the NIR region (650-900 nm), and NIR photons cause less biological damage to cells and tissues (10). Moreover, C5SA itself also has low cytotoxicity (10). We therefore consider that C5S-A is suitable for observing and analyzing viable CSCs.

In this study, we validated the utility of C5S-A for CSC research in breast cancer cell lines. Furthermore, we clarified how long fluorescence remained observable after a single staining and evaluated whether C5S-A was useful for chronological studies in living cells using a drug-resistance experiment.

\section{Materials and Methods}

Cell lines and cell culture. HCC1954, SKBR3, MDAMB453 and MDAMB468 cell lines were obtained from American Type Culture Collection (Manassas, VA, USA). All cells were maintained in RPMI-1640 Media (Thermo Fisher Scientific, Waltham, MA, USA) supplemented with $10 \%$ fetal bovine serum and $1 \%$ penicillin/ streptomycin, and grown at $37^{\circ} \mathrm{C}$ in a humidified atmosphere with $5 \% \mathrm{CO}_{2}$.
ALDEFLUOR and C5S-A staining. The enzymatic activity of ALDH1 was detected using ALDEFLUOR Staining Kit (STEMCELL Technologies, Inc., Vancouver, BC, Canada). Staining was carried out according to the manufacturer's instructions.

For staining with C5S-A (Figure 1) (10), a NIR turn-on fluorescence probe that becomes emissive in the presence of ALDH1A1, the individual breast cancer cell lines were seeded in $100 \mathrm{~mm}$ dishes $\left(3 \times 10^{6} / 10 \mathrm{ml}\right)$ (Falcon, Durham, NC, USA). C5S-A dissolved in dimethyl sulfoxide was diluted with the medium (final concentration: $1 \mu \mathrm{M}$ ) without phenol red or fetal bovine serum and the cells were incubated with the medium at $37^{\circ} \mathrm{C}$ for $30 \mathrm{~min}$.

Flow cytometric analysis and cell sorting. After each breast cancer cell line was stained with ALDEFLUOR or C5S-A by the above methods, flow cytometric analysis and fluorescence-activated cell sorting were performed using a FACSAria III (BD Biosciences, Franklin Lakes, NJ, USA) with a $488 \mathrm{~nm}$ laser and fluorescein isothiocyanate filter for ALDEFLUOR, or a $633 \mathrm{~nm}$ laser and allophycocyanin filter for C5S-A. To determine the population of CD44-positive/CD 24-negative cells, HCC1954 cells were stained for 20 min at $4^{\circ} \mathrm{C}$ with anti-human BV421-conjugated CD44 and phycoerythrin-conjugated CD24 antibodies (BD Biosciences). Isotype-matched antibodies were used as controls.

Fluorescence microscopy imaging. Fluorescence images of each breast cancer cell line were obtained and qualitatively evaluated with a BZ-X700 fluorescence microscope (Keyence, Osaka, Osaka, Japan) with a $10 \times$ objective lens, and a green fluorescent protein filter for ALDEFLUOR analysis and Cy5 filter for C5S-A analysis.

Mammosphere assays. C5S-A-positive and -negative breast cancer cells (HCC1954, SKBR3, MDAMB453 and MDAMB468; 2×104) were sorted by FACS as described above. These cells were then seeded $\left(2 \times 10^{4} / 2 \mathrm{ml}\right)$ in 6-well low-adherence plates (Corning, Corning, NY, USA) and cultured in a MammoCult Human Medium (STEMCELL Technologies, Inc.) supplemented with hydrocortisone stock solution and heparin solution $(0.48 \mu \mathrm{g} / \mathrm{ml}$ and $4.0 \mu \mathrm{g} / \mathrm{ml}$, respectively) (STEMCELL Technologies, Inc.). We then counted the number of mammospheres in nine fields of view in each 6-well plate for 4-5 days.

Transplantation experiments. Sorted C5S-A-positive and -negative breast cancer cells (HCC1954, MDAMB453 and MDAMB468; 
$5 \times 10^{5}$ ) were transplanted into the subcutaneous mammary gland of the inguinal region of two NOD/LtSz-scid/IL2R $\gamma^{-/-}$(NSG) mice for each cell line, purchased from Charles River Laboratories (Wilmington, MA, USA). Tumor growth was measured once a week with calipers. Animal experiments were conducted under a protocol approved by the Kobe University Animal Experimentation Committee (P170805-R2).

Chronological change in area ratio of C5S-A expression during drug treatment. HCC1954 and MDAMB468 breast cancer cells were seeded in a 24 -well plate at a density of $1.0 \times 10^{5}$ cells and allowed to grow for 2 days. They were then stained with C5S-A and cultured with $0.05 \mu \mathrm{M}$ doxorubicin or $0.05 \mu \mathrm{M}$ paclitaxel. Images were captured with a BZ-X700 with a 10× objective lens and Cy5 filter on days 0 and 3 . The area of C5S-A-expressing cells as a ratio to all cells was measured with software, BZ-X Analyzer (Keyence), and the ratio was compared between days 0 and 3 .

Statistical analysis. All statistical analyses were performed using SPSS Statistics version 19.0 (IBM, Chicago, IL, USA). Results are presented as mean values \pm SEM (standard error of the mean) from triplicate wells, and all data were representative of at least two independent experiments. Statistical analysis was performed using two-tailed Student's $t$-tests and values of $p<0.05$ were considered statistically significant.

\section{Results}

Concordance between conventional staining for stem cell markers and C5S-A. To evaluate concordance between staining by ALDEFLUOR and that by C5S-A, we sorted breast cancer cells in ALDEFLOUR-positive and -negative areas by FACS (Figure 2A). Sorted ALDH-positive cells were clearly stained by C5S-A. In contrast, ALDH-negative cells were not stained by C5S-A (Figure 2A).

We investigated the association of CD44-positive/CD24negative cells, a known breast CSC population, with C5S-Apositive cells (Figure 2B). The HCC1954 cell line, which has a CD44-positive/CD24-negative cell population, was selected for this study. Sorted CD44-positive/CD24-negative cells were clearly stained with C5S-A, while cells of other sorted populations were not (Figure 2B). Additionally, we confirmed that CD44-positive/CD24-negative cells formed mammospheres (Figure 2B).

Tumorigenic properties of C5S-A-positive breast cancer cells. To evaluate the tumorigenic properties of C5S-Apositive cells in vitro, $2 \times 10^{4} \mathrm{C} 5 \mathrm{~S}-\mathrm{A}$-positive and negative cells from each cell line (HCC1954, SKBR3, MDAMB453 and MDAMB468) were sorted by FACS and submitted to mammosphere assay (Figure 3A). In all cell lines, C5S-Apositive cells formed significantly more mammospheres than C5S-A-negative cells (Figure 3A and B).

To test the tumorigenicity of C5S-A-positive breast cancer cells in vivo, we transplanted C5S-A-positive and -negative cells (HCC1954, MDAMB453 and MDAMB468) into the inguinal mammary gland of NSG mice. For all cell lines, C5S-A-positive cells generated a tumor (Figure 3C). In contrast, C5S-A-negative cells failed to generate tumors (Figure 3C).

Evaluation of chemotherapy resistance over time by C5S-A. Firstly, to evaluate how long fluorescence from a single staining remained observable, we cultured SKBR3 breast cancer cells for 5 days after ALDEFLUOR or C5S-A staining. Although the luminescence of ALDEFLUOR disappeared after 1 day, that of C5S-A lasted for at least 3 days (Figure 4).

Next, we chronologically evaluated the change in C5S-Apositive cell area after 3 days of treatment of HCC1954 and MDAMB468 cell lines with doxorubicin or paclitaxel. The C5SA-positive cell area was significantly increased after treatment in both cell lines (Figure 5), indicating that non-CSCs were killed by chemotherapy and that the ratio of CSCs increased.

\section{Discussion}

In this study, we demonstrated that $\mathrm{C} 5 \mathrm{~S}-\mathrm{A}$ is suitable for use in $\mathrm{CSC}$ research in breast cancer. Moreover, we also demonstrated that $\mathrm{C} 5 \mathrm{~S}-\mathrm{A}$ has a long luminescence duration, more than 3 days, and is thus suitable for observing and analyzing viable cells over time. Indeed, we were able to use this characteristic of C5S-A to chronologically evaluate drug resistance to cytotoxic agents, which is a feature of CSCs. Interestingly, recent studies have also shown that CSCs have plasticity and a dynamic nature over time $(11,12)$. Chronological imaging of living cells is a therefore critical component of research into CSC behavior, and we believe that C5S-A will prove useful in various areas requiring time-lapse imaging.

C5S-A-positive cells in our study showed very strong tumorigenic potential. In humans, 19 different ALDH isoforms have been identified (9). Although all of these are associated with reducing oxidative stress and protecting cells from damage, it is unclear whether they are all associated with the characteristics of CSCs (9). Among these isoforms, ALDH1A1 has been well researched as the key ALDH isozyme linked to the CSC population (9). Importantly, this isoform has been reported to provide drug resistance to agents cytotoxic to CSCs (13). One group reported that abundant expression of ALDH1A1 was associated with expression of P-glycoprotein (breast cancer-resistance protein) (14). These findings have increased the focus on ALDH1A1 in cancer research, and better technologies for identifying and isolating ALDH1A1positive cells are required. Although ALDEFLUOR is a nonselective probe for some ALDH isoforms (15), we demonstrated that C5S-A shows high selectivity for ALDH1A1 (10). In this study, C5S-A-positive cells exhibited characteristics of not only tumorgenicity but also drug resistance. We consider that this ALDH1A1-specific probe will prove useful in researching these characteristics in detail. 


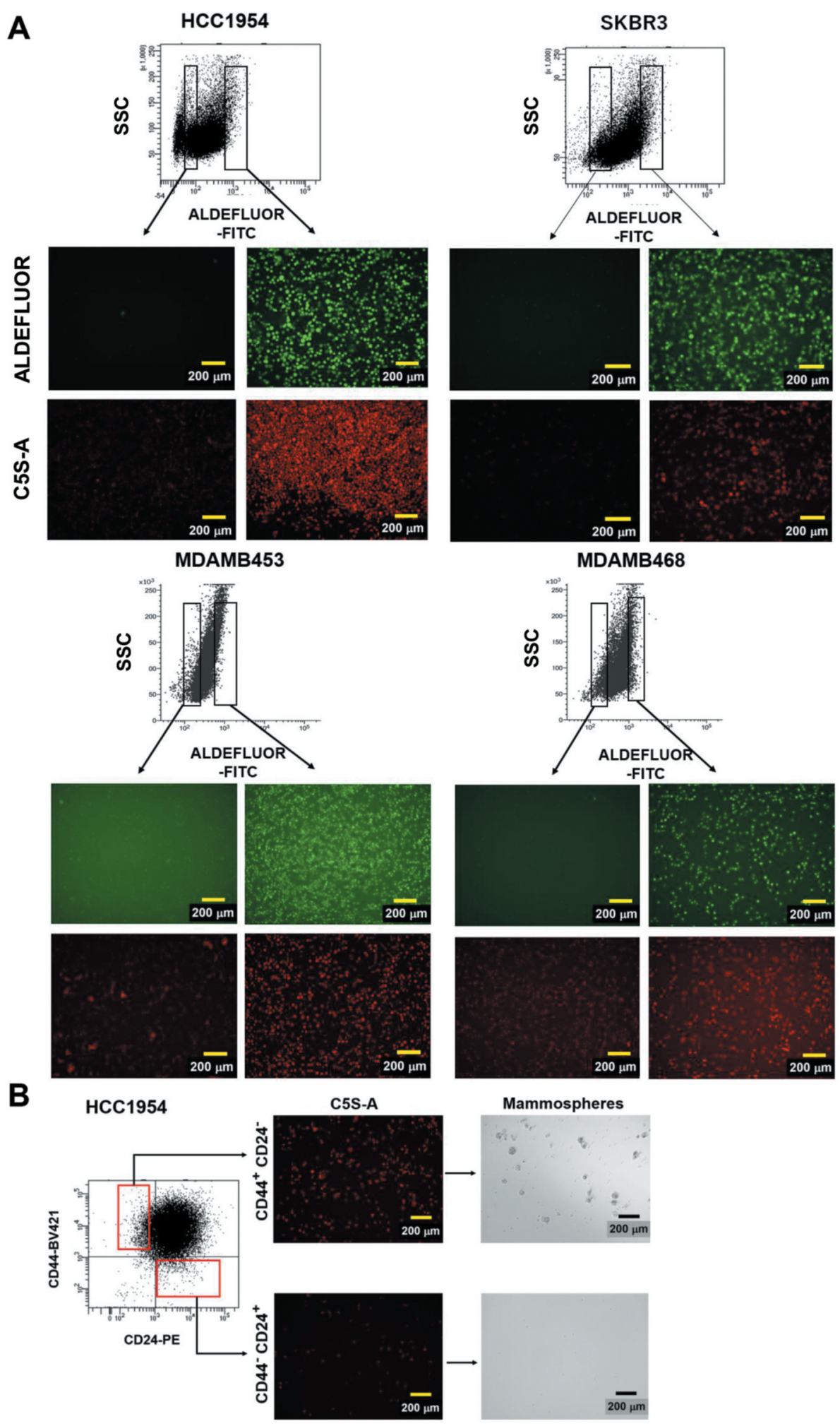

Figure 2. Images of fluorescence in cell lines treated with ALDEFLUOR and C5S-A and association of C5S-A with known stem cell populations. A: ALDEFLUOR-positive and -negative cells were stained with C5S-A and photographed under a fluorescence microscope. Strong green and red fluorescence was observed in ALDEFLUOR-positive cells and C5S-A-positive cells, respectively. Scale bar: $200 \mu$ m. B: HCC1954 cells were sorted by fluorescence-activated cell sorting based on the expression of the cancer stem cell markers CD44 and CD24 and each cell population was stained with C5S-A. Red fluorescence was observed in CD44-positive/CD24-negative cells, and mammospheres were formed. Scale bar: $200 \mu \mathrm{m}$. 
A

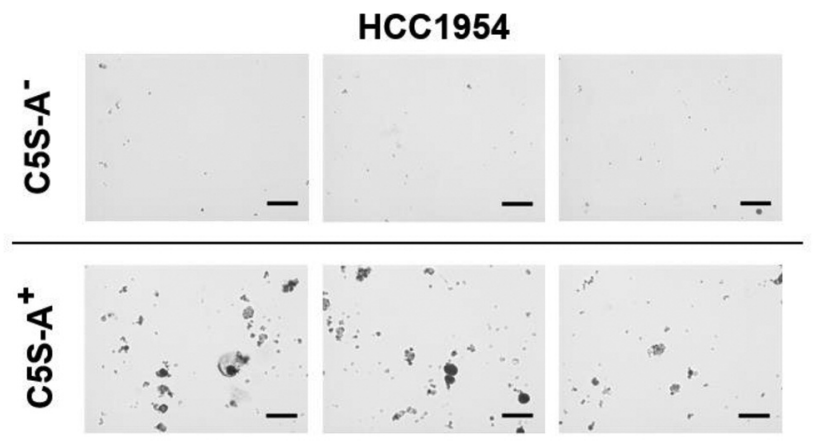

SKBR3
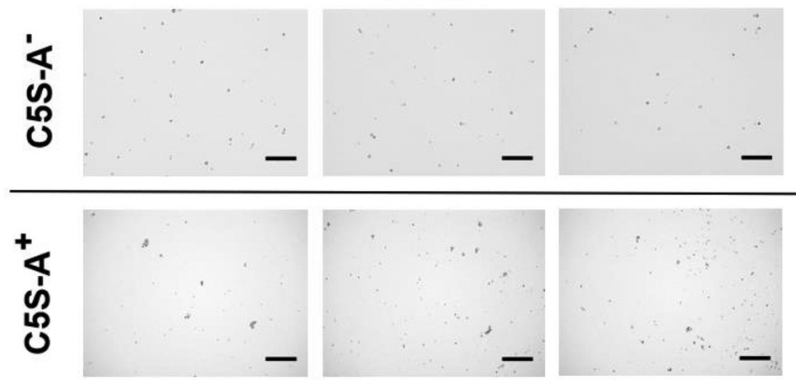

B

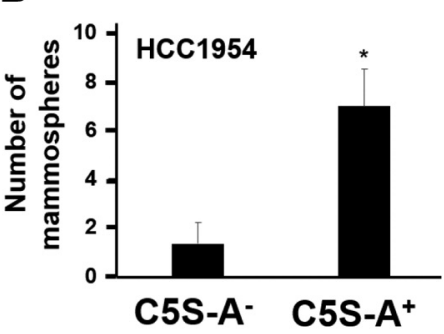

C
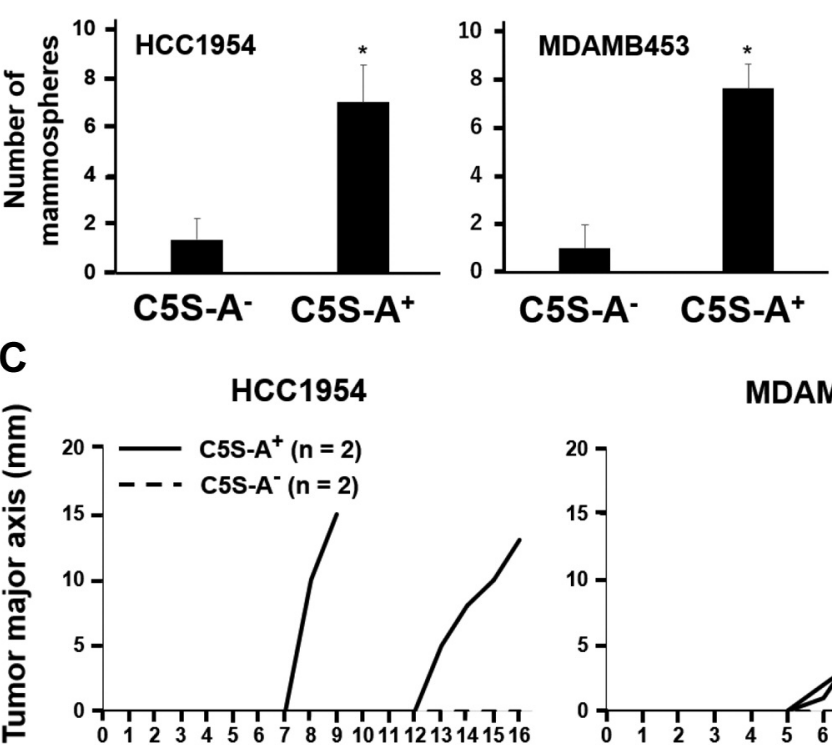
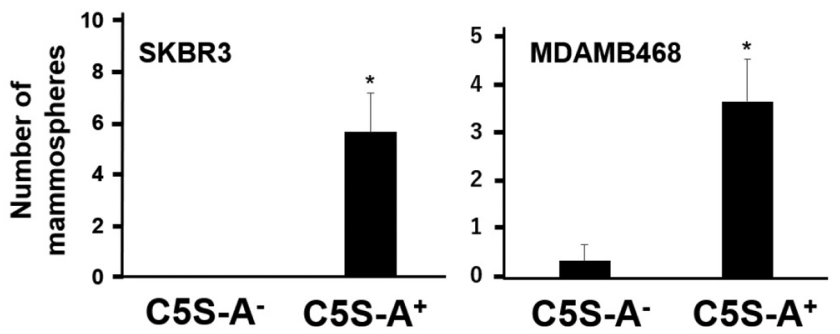

MDAMB453

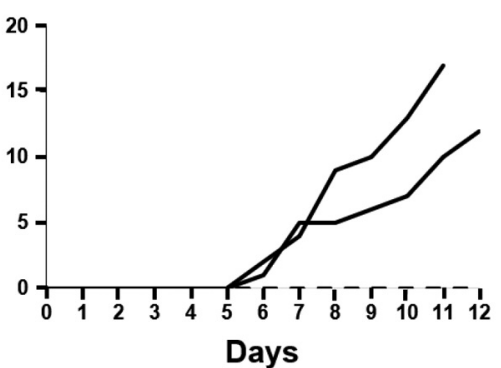

MDAMB453

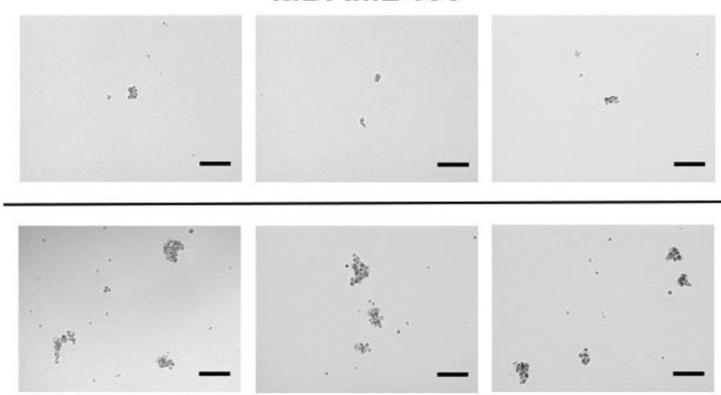

MDAMB468
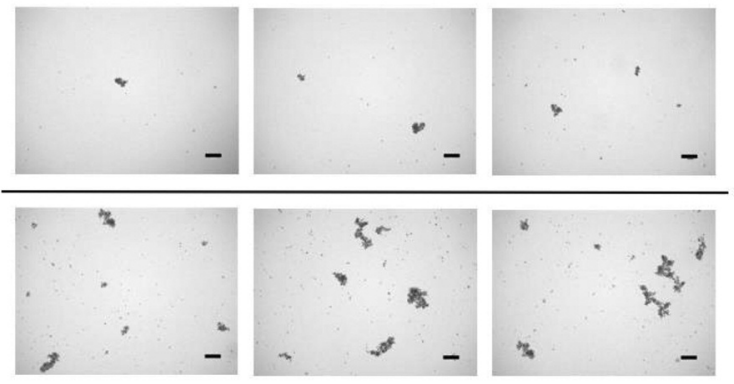

MDAMB468

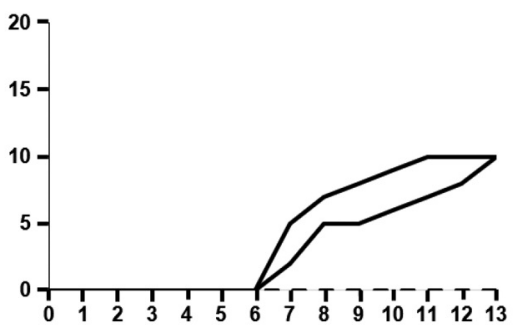

Figure 3. Stem cell properties of C5S-A-positive cells. A: C5S-A-positive and-negative cells were separated by fluorescence-activated cell sorting and subjected to mammosphere formation assay. Mammospheres were found to be formed by C5S-A-positive cells. Scale bar: $200 \mu m$. B: C5S-A-positive cells were highly capable of forming mammospheres. Data are presented as the mean $\pm S E M(n=3$ individual experiments). $*$ Significantly different at $p<0.05$. $C$ : Breast cancer cell lines were transplanted into inguinal mammary glands of female NOD/LtSz-scid/IL2R ${ }^{-1-}$ (NSG) mice. Tumor growth curves were plotted for each population (C5S-A-positive, C5S-A-negative). Tumor formation was observed in the group injected with C5S-A-positive cells.

In conclusion, we showed that $\mathrm{C} 5 \mathrm{~S}-\mathrm{A}$ is suitable for CSC research using breast cancer cell lines, and confirmed its utility in observing cells over time. We are currently planning further studies on the application of this probe to in vivo imaging and clinical use.

\section{Conflicts of Interest}

Hironobu Minami has received research grants and honoraria from Bayer Yakuhin, Boehringer Ingelheim, Bristol-Myers Squibb, Chugai Pharmaceutical, DaiichiSankyo, Eisai, Kyowa-Kirin, Merck 


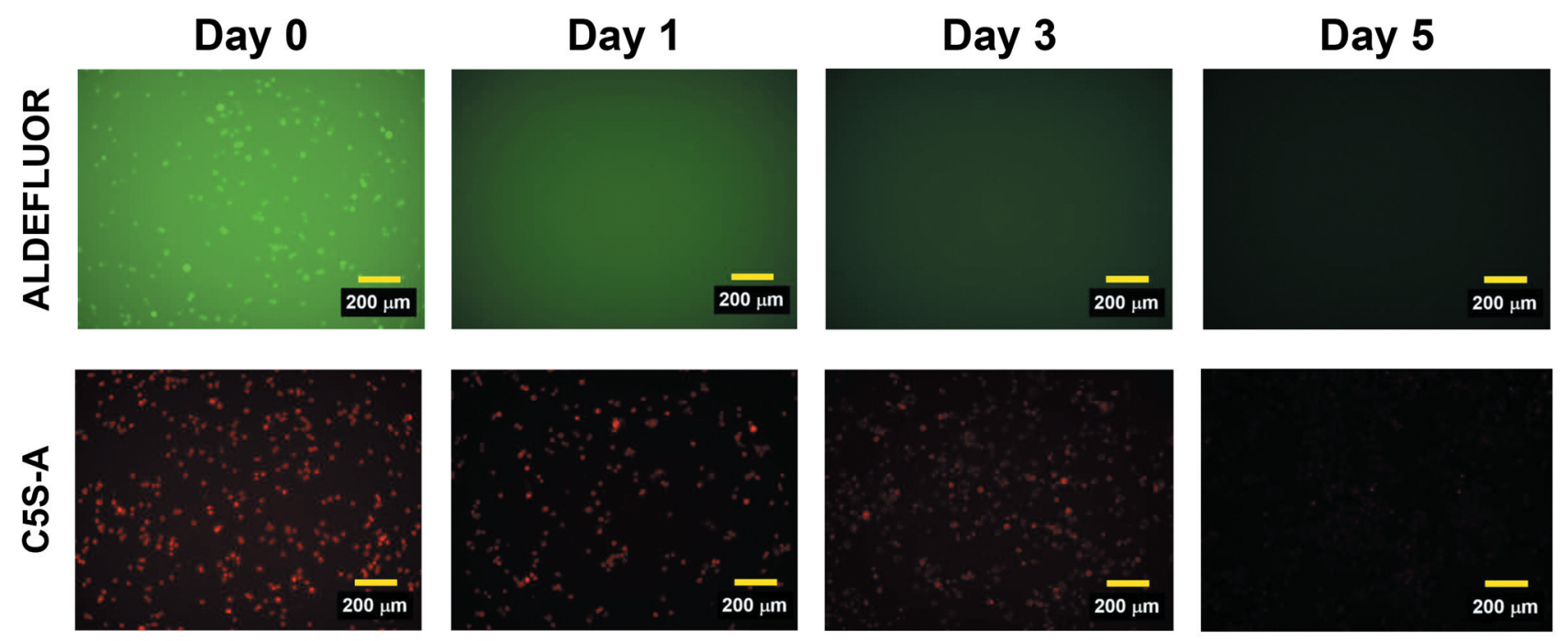

Figure 4. Changes over time in fluorescence of ALDEFLUOR and C5S-A. SKBR3 cells were stained with ALDEFLUOR and C5S-A and photographed with a fluorescence microscope to determine the duration of luminescence. The fluorescence of ALDEFLUOR disappeared within 1 day, whereas that of C5S-A persisted for 3 days. Scale bar: $200 \mu \mathrm{m}$.
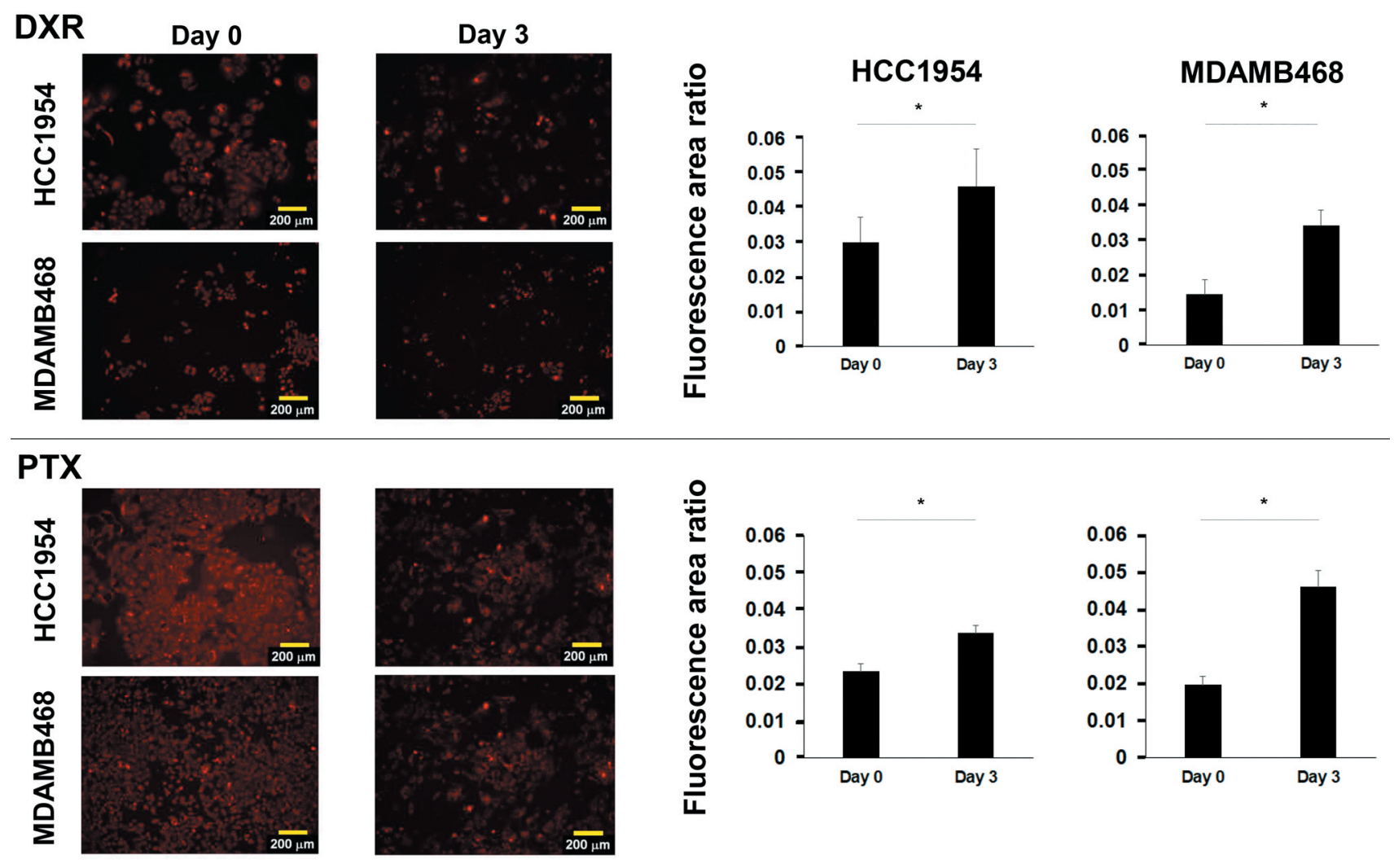

Figure 5. Evaluation of resistance of HCC1954 and MDAMB468 cells to anticancer drugs by ALDH1A1-positive cells using C5S-A. Fluorescence images were taken before and 3 days after doxorubicin or paclitaxel administration. C5S-A-positive cells emitted red fluorescence. Scale bar: 200 $\mu \mathrm{m}$. The ratio of C5S-A-positive cells (area of C5S-A-positive cells per total cell area) before and after drug administration are shown on the right. The C5S-A-positive cell area ratio was higher after treatment. Data are presented as the mean $\pm S E M(n=3$ individual experiments). *Significantly different at $p<0.05$. 
Serono, MSD, Novartis, Ono Pharmaceutical, Pfizer, Sanofi, Takeda Pharmaceutical, Taiho Pharmaceutical, and Eli Lilly; research grants from Asahi-Kasei Pharma, Astellas Pharma, Nippon Shinyaku, Yakult Honsha, CSL, Behring, and Nippon Kayaku; and honoraria from Celgene, Otsuka Pharmaceutical, Shire Japan, Genomic Health, and Abbvie. Naomi Kiyota reports grants from BristolMyers Squibb and Ono Pharmaceutical during the conduct of the study; grants from Astra-Zeneca and Roche Pharmaceuticals outside the submitted work; and honoraria from Ono Pharmaceutical, Bristol-Myers Squibb, Merck Biopharma, Astra-Zeneca, Merck Sharp \& Dohme, Eisai, and Bayer. The other Authors declare no conflicts of interest.

\section{Authors' Contributions}

AO and YF: Conception, study design, data collection, analysis, interpretation, drafting and writing of the article; MO and KM: conception, study design, data collection, analysis, interpretation, drafting; RT, HS, YN, MN and YI: conception, study, analysis, interpretation; TK, NK, KO and HT: conception, study design; HM: supervision of the project. All Authors read and approved the final version of the article.

\section{References}

1 Reya T, Morrison SJ, Clarke MF and Weissman IL: Stem cells, cancer, and cancer stem cells. Nature 414(6859): 105-111, 2001 PMID: 11689955 . DOI: $10.1038 / 35102167$

2 Zheng Q, Zhang M, Zhou F, Zhang L and Meng X: The breast cancer stem cells traits and drug resistance. Front Pharmacol 11: 599965, 2021. PMID: 33584277. DOI: 10.3389/fphar.2020.599965

3 Ginestier C, Hur MH, Charafe-Jauffret E, Monville F, Dutcher J, Brown M, Jacquemier J, Viens P, Kleer CG, Liu S, Schott A, Hayes D, Birnbaum D, Wicha MS and Dontu G: ALDH1 is a marker of normal and malignant human mammary stem cells and a predictor of poor clinical outcome. Cell Stem Cell 1(5): 555-567, 2007. PMID: 18371393. DOI: 10.1016/j.stem.2007.08.014

4 Bettinardi V, Picchio M, Di Muzio N and Gilardi MC: Motion management in positron emission tomography/computed tomography for radiation treatment planning. Semin Nucl Med 42(5): 289-307, 2012. PMID: 22840595. DOI: 10.1053/ j.semnuclmed.2012.04.001

5 Chen Y, Thompson DC, Koppaka V, Jester JV and Vasiliou V: Ocular aldehyde dehydrogenases: protection against ultraviolet damage and maintenance of transparency for vision. Prog Retin Eye Res 33: 28-39, 2013. PMID: 23098688. DOI: 10.1016/j.preteyeres. 2012.10.001

6 Charafe-Jauffret E, Ginestier C, Iovino F, Tarpin C, Diebel M, Esterni B, Houvenaeghel G, Extra JM, Bertucci F, Jacquemier J, Xerri L, Dontu G, Stassi G, Xiao Y, Barsky SH, Birnbaum D, Viens P and Wicha MS: Aldehyde dehydrogenase 1-positive cancer stem cells mediate metastasis and poor clinical outcome in inflammatory breast cancer. Clin Cancer Res 16(1): 45-55, 2010. PMID: 20028757. DOI: 10.1158/1078-0432.CCR-09-1630
7 Croker AK, Goodale D, Chu J, Postenka C, Hedley BD, Hess DA and Allan AL: High aldehyde dehydrogenase and expression of cancer stem cell markers selects for breast cancer cells with enhanced malignant and metastatic ability. J Cell Mol Med 13(8B): 2236-2252, 2009. PMID: 18681906. DOI: 10.1111/j.15824934.2008.00455.x

8 Yoshida A, Davé V, Han H and Scanlon KJ: Enhanced transcription of the cytosolic ALDH gene in cyclophosphamide resistant human carcinoma cells. Adv Exp Med Biol 328: 63-72, 1993. PMID: 8493941. DOI: 10.1007/978-1-4615-2904-0_8

9 Tomita H, Tanaka K, Tanaka $\mathrm{T}$ and Hara A: Aldehyde dehydrogenase $1 \mathrm{~A} 1$ in stem cells and cancer. Oncotarget 7(10): 11018-11032, 2016. PMID: 26783961. DOI: 10.18632/oncotarget. 6920

10 Oe M, Miki K, Ueda Y, Mori Y, Okamoto A, Funakoshi Y, Minami $\mathrm{H}$ and Ohe $\mathrm{K}$ : Deep-red/near-infrared turn-on fluorescence probes for aldehyde dehydrogenase 1A1 in cancer stem cells. ACS Sens 6(9): 3320-3329, 2021. PMID: 34445866. DOI: $10.1021 /$ acssensors.1c01136

11 Thankamony AP, Saxena K, Murali R, Jolly MK and Nair R: Cancer stem cell plasticity - a deadly deal. Front Mol Biosci 7: 79, 2020. PMID: 32426371. DOI: 10.3389/fmolb.2020.00079

12 Kreso A and Dick JE: Evolution of the cancer stem cell model. Cell Stem Cell 14(3): 275-291, 2014. PMID: 24607403. DOI: 10.1016/j.stem.2014.02.006

13 Koppaka V, Thompson DC, Chen Y, Ellermann M, Nicolaou KC, Juvonen RO, Petersen D, Deitrich RA, Hurley TD and Vasiliou V: Aldehyde dehydrogenase inhibitors: a comprehensive review of the pharmacology, mechanism of action, substrate specificity, and clinical application. Pharmacol Rev 64(3): 520539, 2012. PMID: 22544865. DOI: 10.1124/pr.111.005538

14 Januchowski R, Wojtowicz K, Sterzyfska K, Sosifska P, Andrzejewska M, Zawierucha P, Nowicki $\mathrm{M}$ and Zabel M: Inhibition of ALDH1A1 activity decreases expression of drug transporters and reduces chemotherapy resistance in ovarian cancer cell lines. Int J Biochem Cell Biol 78: 248-259, 2016. PMID: 27443528. DOI: 10.1016/j.biocel.2016.07.017

15 Zhou L, Sheng D, Wang D, Ma W, Deng Q, Deng L and Liu S: Identification of cancer-type specific expression patterns for active aldehyde dehydrogenase (ALDH) isoforms in ALDEFLUOR assay. Cell Biol Toxicol 35(2): 161-177, 2019. PMID: 30220009. DOI: 10.1007/s10565-018-9444-y

Received January 6, 2022

Revised January 31, 2022

Accepted February 7, 2022 